\title{
Nonexistence Results of Semilinear Elliptic Equations Coupled with the Chern-Simons Gauge Field
}

\author{
Hyungjin Huh \\ Department of Mathematics, Chung-Ang University, Seoul 156-756, Republic of Korea \\ Correspondence should be addressed to Hyungjin Huh; huh@cau.ac.kr \\ Received 1 November 2012; Accepted 15 January 2013 \\ Academic Editor: Khalil Ezzinbi
}

Copyright (C) 2013 Hyungjin Huh. This is an open access article distributed under the Creative Commons Attribution License, which permits unrestricted use, distribution, and reproduction in any medium, provided the original work is properly cited.

We discuss the nonexistence of nontrivial solutions for the Chern-Simons-Higgs and Chern-Simons-Schrödinger equations. The Derrick-Pohozaev type identities are derived to prove it.

\section{Introduction and Main Results}

In this paper, we are concerned with the nonexistence of nontrivial solutions to some elliptic equations coupled with Chern-Simons gauge field. More precisely, let us first consider the following system:

$$
\begin{gathered}
-\left(\omega+A_{0}\right)^{2} \phi-D_{1} D_{1} \phi-D_{2} D_{2} \phi+V^{\prime}\left(|\phi|^{2}\right) \phi=0, \\
\partial_{1} A_{0}=-\operatorname{Im}\left(\bar{\phi} D_{2} \phi\right), \\
\partial_{2} A_{0}=\operatorname{Im}\left(\bar{\phi} D_{1} \phi\right), \\
\partial_{1} A_{2}-\partial_{2} A_{1}=-\left(\omega+A_{0}\right)|\phi|^{2},
\end{gathered}
$$

which is derived from the system (5) with stationary solution ansatz $\psi(t, x)=e^{i \omega t} \phi(x), \phi \in \mathbb{C}$, and $A_{\mu}(t, x)=A_{\mu}(x)$ for $\mu=0,1,2$. Consider

$$
\begin{gathered}
D_{0} D_{0} \psi-\left(D_{1} D_{1}+D_{2} D_{2}\right) \psi+V^{\prime}\left(|\psi|^{2}\right) \psi=0, \\
\partial_{0} A_{1}-\partial_{1} A_{0}=\operatorname{Im}\left(\bar{\psi} D_{2} \psi\right), \\
\partial_{0} A_{2}-\partial_{2} A_{0}=-\operatorname{Im}\left(\bar{\psi} D_{1} \psi\right), \\
\partial_{1} A_{2}-\partial_{2} A_{1}=-\operatorname{Im}\left(\bar{\psi} D_{0} \psi\right),
\end{gathered}
$$

where $\partial_{0}=\partial / \partial t, \partial_{1}=\partial / \partial x_{1}, \partial_{2}=\partial / \partial x_{2}$ for $\left(t, x_{1}, x_{2}\right) \epsilon$ $\mathbb{R}^{1+2}, \psi: \mathbb{R}^{1+2} \rightarrow \mathbb{C}$ is the complex scalar field, $A_{\mu}: \mathbb{R}^{1+2} \rightarrow$ $\mathbb{R}$ is the gauge field, $D_{\mu}=\partial_{\mu}+i A_{\mu}$ is the covariant derivative for $\mu=0,1,2$, and $i$ denotes the imaginary unit.
The Chern-Simons-Higgs system in (5) was introduced in $[1,2]$ to deal with the electromagnetic phenomena in planar domain such as fractional quantum Hall effect or high temperature superconductivity. The system in (5) has the conservation of the total energy

$$
E(t)=\int_{\mathbb{R}^{2}} \sum_{\alpha=0}^{2}\left|D_{\alpha} \psi(t, x)\right|^{2}+V\left(|\psi(t, x)|^{2}\right) d x=E(0) .
$$

The special case with a self-dual potential $V\left(|\phi|^{2}\right)=(1 / 4)$ $|\phi|^{2}\left(|\phi|^{2}-1\right)^{2}$ has received much attention and has been studied by several authors, where one can derive the following system of first-order equations called self-dual equations (see $[1,2])$

$$
\begin{gathered}
D_{1} \phi-i D_{2} \phi=0, \\
\partial_{1} A_{2}-\partial_{2} A_{1}+\frac{1}{2}|\phi|^{2}\left(|\phi|^{2}-1\right)=0, \\
\omega+A_{0}-\frac{i}{2}\left(|\phi|^{2}-1\right) \phi=0 .
\end{gathered}
$$

We note that solutions to the self-dual equations (7) provide solutions to (1)-(4). For the self-dual potential $V\left(|\phi|^{2}\right)=$ $(1 / 4)|\phi|^{2}\left(|\phi|^{2}-1\right)^{2}$, there are two possible boundary conditions to make the energy finite; either $|\phi| \rightarrow 1$ or $|\phi| \rightarrow 0$ as $|x| \rightarrow \infty$. The former boundary condition is called "topological" while the latter "non-topological." A lot 
of works have been done for the existence of solutions to the self-dual system [3-7]. Some existence results for the nonselfdual Chern-Simons-Higgs equations with the topological boundary condtion have been proved in [8-10]. From the mathematical point of view, it is meaningful to study existence and nonexistence of nontrivial solutions under various conditions on $V$. In this paper, we are concerned with the nonexistence of the non-trivial solution to (1)-(4) with the non-topological boundary condtion. The following is our first result.

Theorem 1. Let $\left(\phi, A_{0}, A_{1}, A_{2}\right)$ be a classical solution of (1)(4) such that $\phi \in H^{1}\left(\mathbb{R}^{2}\right)$ and $A_{0} \in L^{p}\left(\mathbb{R}^{2}\right), A_{1}, A_{2} \in L^{q}\left(\mathbb{R}^{2}\right)$ for any $2<p, q \leq \infty$. Let $V: \mathbb{R} \rightarrow \mathbb{R}$ be a $C^{1}$ function such that $V(0)=0, \inf \{x>0 \mid V(x) \neq 0\}=0$ and $V\left(|\phi|^{2}\right)$, $|\phi|^{2} V^{\prime}\left(|\phi|^{2}\right) \in L^{1}\left(\mathbb{R}^{2}\right)$. Assume that

$$
d V^{\prime}(s) s-V(s) \geq 0 \quad \forall s \geq 0,
$$

where $0 \leq d \leq 1$ is a constant. Then, one has $\phi \equiv 0$.

The proof is based on the following Derrick-Pohozaev type identity for (1)-(4):

$$
\begin{gathered}
\int_{\mathbb{R}^{2}} d\left(\left|D_{1} \phi\right|^{2}+\left|D_{2} \phi\right|^{2}\right)+(1-d)\left(\omega+A_{0}\right)^{2}|\phi|^{2} \\
+d V^{\prime}\left(|\phi|^{2}\right)|\phi|^{2}-V\left(|\phi|^{2}\right) d x=0 .
\end{gathered}
$$

As a typical example, we consider $V\left(|\phi|^{2}\right)=\alpha|\phi|^{6}+\beta|\phi|^{4}$. Then it is easy to check that $d V^{\prime}(s) s-V(s)=\alpha(3 d-1) s^{3}+$ $\beta(2 d-1) s^{2}$. If one of the following conditions is satisfied, then we have $\phi \equiv 0$.

(1) For $\alpha>0, \beta>0$, we take $1 / 2<d<1$.

(2) For $\alpha>0, \beta<0$, we take $1 / 3<d<1 / 2$.

(3) For $\alpha<0, \beta<0$, we take $0<d<1 / 3$.

Note that for the self-dual potential $V\left(|\phi|^{2}\right)=(1 / 4)|\phi|^{2}\left(|\phi|^{2}-\right.$ $1)^{2}$, we have

$$
d V^{\prime}(s) s-V(s)=\frac{3 d-1}{4} s^{3}-\frac{2 d-1}{2} s^{2}+\frac{d-1}{4} s
$$

which is not nonnegative for $s \geq 0$.

The following Chern-Simons gauged Schrödinger system was proposed in [11] when the second quantized $N$ body anyon problem is considered

$$
\begin{gathered}
i D_{0} \psi+\left(D_{1} D_{1}+D_{2} D_{2}\right) \psi-V^{\prime}\left(|\phi|^{2}\right) \phi=0, \\
\partial_{0} A_{1}-\partial_{1} A_{0}=-\operatorname{Im}\left(\bar{\psi} D_{2} \psi\right), \\
\partial_{0} A_{2}-\partial_{2} A_{0}=\operatorname{Im}\left(\bar{\psi} D_{1} \psi\right) \\
\partial_{1} A_{2}-\partial_{2} A_{1}=-\frac{1}{2}|\psi|^{2}
\end{gathered}
$$

With the stationary solution ansatz $\psi(t, x)=e^{i \omega t} \phi(x), \phi \in \mathbb{C}$ and $A_{\mu}(t, x)=A_{\mu}(x)$ for $\mu=0,1,2$, we arrive at

$$
\begin{gathered}
\left(\omega+A_{0}\right) \phi-D_{1} D_{1} \phi-D_{2} D_{2} \phi+V^{\prime}\left(|\phi|^{2}\right) \phi=0, \\
\partial_{1} A_{0}=\operatorname{Im}\left(\bar{\phi} D_{2} \phi\right), \\
\partial_{2} A_{0}=-\operatorname{Im}\left(\bar{\phi} D_{1} \phi\right), \\
\partial_{1} A_{2}-\partial_{2} A_{1}=-\frac{1}{2}|\phi|^{2} .
\end{gathered}
$$

In the special case with the potential $V\left(|\phi|^{2}\right)=-(1 / 2)|\phi|^{4}$, we can derive the following self dual equations [11-13]

$$
\begin{gathered}
D_{1} \phi+i D_{2} \phi=0, \\
\omega+A_{0}-\frac{1}{2}|\phi|^{2}=0, \\
\partial_{1} A_{2}-\partial_{2} A_{1}+\frac{1}{2}|\phi|^{2}=0 .
\end{gathered}
$$

Note that solutions to the self-dual system (16) provide solutions to (12)-(15). The self-dual equations (16) can be transformed into the Liouville equation, an integrable equation whose solutions are explicitly known.

For the nonself-dual potential of the form $V\left(|\phi|^{2}\right)=$ $-\lambda|\phi|^{p}(\lambda>0, p>2)$, some existence and nonexistence results have been studied in $[14,15]$ under the condition of the radially symmetric solution $\psi(t, x)=e^{i \omega t} u(|x|)(u \in \mathbb{R})$. We prove the following nonexistence result, under various conditions on $V$, for (12)-(15).

Theorem 2. Let $\left(\phi, A_{0}, A_{1}, A_{2}\right)$ be a classical solution of (1)(4) such that $\phi \in H^{1}\left(\mathbb{R}^{2}\right), A_{0} \in L^{p}\left(\mathbb{R}^{2}\right)$ and $A_{1}, A_{2} \in L^{q}\left(\mathbb{R}^{2}\right)$ for $1<p \leq \infty$ and $2<q \leq \infty$. One also assumes that $V$ : $\mathbb{R} \rightarrow \mathbb{R}$ is a $C^{1}$ function such that $V(0)=0$ and $V\left(|\phi|^{2}\right)$, $|\phi|^{2} V^{\prime}\left(|\phi|^{2}\right) \in L^{1}\left(\mathbb{R}^{2}\right)$.

(1) If the potential $V$ satisfies

$$
V^{\prime}(s) s-V(s) \geq 0 \quad \forall s \geq 0
$$

then one has $\phi \equiv 0$.

(2) Suppose that $\phi$ is a real-valued function; that is, $\phi(x)=$ $u(x) \in \mathbb{R}$ and $1 / p+1 / q=1$ for $2<q<\infty$. If the potential $V$ satisfies, for a constant $h \geq 2 / 3$,

$$
\omega(h-1) s+h V^{\prime}(s) s-V(s) \geq 0 \quad \forall s \geq 0,
$$

then one has $u \equiv 0$.

The proof is based on the Derrick-Pohozaev type identities (40) and (45) for (12)-(15).

Example 3. For the static solution $(\omega=0)$, we consider the potential $V(s)=s^{3}-s^{2}-s$. Then, taking $h=2 / 3$, we can check

$$
\frac{2}{3} V^{\prime}\left(|u|^{2}\right)|u|^{2}-V\left(|u|^{2}\right)=|u|^{6}-\frac{1}{3}|u|^{4}+\frac{1}{3}|u|^{2} \geq 0 .
$$


However we have, for the complex solution,

$$
V^{\prime}\left(|\phi|^{2}\right)|\phi|^{2}-V\left(|\phi|^{2}\right)=2|\phi|^{6}-|\phi|^{4}
$$

which is not nonnegative.

The paper is organized as follows. In Section 2, we prove Theorem 1 by deriving Derrick-Pohozaev type identity. Theorem 2 is proved in Section 3. We conclude this section by giving a few notations.

(i) $H^{1}\left(\mathbb{R}^{2}\right)$ denotes the usual Sobolev space $W^{1,2}\left(\mathbb{R}^{2}\right)$.

(ii) $B_{R}:=\left\{x \in \mathbb{R}^{2}|| x \mid \leq R\right\}$ and $\partial B_{R}:=\left\{x \in \mathbb{R}^{2}|| x \mid=\right.$ $R\}$.

(iii) $d \sigma_{R}:=$ the surface measure on $\partial B_{R}$.

\section{Proof of Theorem 1}

We apply Derrick-Pohozaev argument to derive the identity (9) which prove Theorem 1 . From now on, we adopt the summation convention for repeated indices.

Suppose that $\left(\phi, A_{0}, A_{1}, A_{2}\right)$ is a solution of (1)-(4). Multiplying (1) by $x_{k} \overline{D_{k} \phi}$ and integrating over $B_{R}$, we obtain

$$
\begin{gathered}
-\int_{B_{R}}\left(\omega+A_{0}\right)^{2} \phi x_{k} \overline{D_{k} \phi} d x-\int_{B_{R}} D_{j} D_{j} \phi x_{k} \overline{D_{k} \phi} d x \\
+\int_{B_{R}} V^{\prime}\left(|\phi|^{2}\right) \phi x_{k} \overline{D_{k} \phi} d x=0 .
\end{gathered}
$$

Now we set

$$
\begin{gathered}
\mathrm{I}=\int_{B_{R}}\left(\omega+A_{0}\right)^{2} \phi x_{k} \overline{D_{k} \phi} d x, \\
\mathrm{II}=\int_{B_{R}} D_{j} D_{j} \phi x_{k} \overline{D_{k} \phi} d x, \\
\mathrm{III}=\int_{B_{R}} V^{\prime}\left(|\phi|^{2}\right) \phi x_{k} \overline{D_{k} \phi} d x .
\end{gathered}
$$

Then, integrating by parts and taking real parts, we have

$$
\begin{aligned}
\operatorname{Re}\{\mathrm{I}\}= & \int_{\partial B_{R}} \frac{R}{2}\left(\omega+A_{0}\right)^{2}|\phi|^{2} d \sigma_{x} \\
& -\int_{B_{R}}\left(\omega+A_{0}\right)^{2}|\phi|^{2} d x \\
& -\int_{B_{R}} \omega \partial_{j} A_{0} x_{j}|\phi|^{2} d x \\
& -\int_{B_{R}} \frac{1}{2} x_{j} \partial_{j}\left(A_{0}^{2}\right)|\phi|^{2} d x,
\end{aligned}
$$

$\operatorname{Re}\{\mathrm{III}\}=\int_{\partial B_{R}} \frac{R}{2} V\left(|\phi|^{2}\right) d \sigma_{x}-\int_{B_{R}} V\left(|\phi|^{2}\right) d x$.
For II, we have

$$
\begin{aligned}
\mathrm{II}= & \int_{B_{R}} \partial_{j}\left(x_{k} D_{j} \phi \overline{D_{k} \phi}\right)-\left|D_{j} \phi\right|^{2}-x_{k} D_{j} \phi \overline{D_{j} D_{k} \phi} d x \\
= & \int_{\partial B_{R}} \frac{x_{j} x_{k}}{R} D_{j} \phi \overline{D_{k} \phi} d \sigma_{R} \\
& -\int_{B_{R}}\left|D_{j} \phi\right|^{2} d x-\int_{B_{R}} x_{k} D_{j} \phi \overline{\left(D_{k} D_{j} \phi+i F_{j k} \phi\right)} d x,
\end{aligned}
$$

where we used the notation $F_{j k}=\partial_{j} A_{k}-\partial_{k} A_{j}$ and the following identity:

$$
D_{j} D_{k} \phi=D_{k} D_{j} \phi+i F_{j k} \phi .
$$

Taking the real parts and integrating by parts, we obtain

$$
\begin{aligned}
\operatorname{Re}\{\mathrm{II}\}= & \int_{\partial B_{R}} \frac{x_{j} x_{k}}{R} D_{j} \phi \overline{D_{k} \phi} d \sigma_{R}-\int_{B_{R}}\left|D_{j} \phi\right|^{2} d x \\
& -\int_{B_{R}} \frac{1}{2} x_{k} \partial_{k}\left(\left|D_{j} \phi\right|^{2}\right) d x \\
& -\int_{B_{R}} x_{k} F_{j k} \operatorname{Im}\left(\bar{\phi} D_{j} \phi\right) d x \\
= & \int_{\partial B_{R}} \frac{x_{j} x_{k}}{R} D_{j} \phi \overline{D_{k} \phi} d \sigma_{R}-\int_{\partial B_{R}} \frac{R}{2}\left|D_{j} \phi\right|^{2} d \sigma_{R} \\
& +\int_{B_{R}} \frac{1}{2}|\phi|^{2} x_{j} \partial_{j}\left(A_{0}^{2}\right) d x+\int_{B_{R}} \omega|\phi|^{2} x_{j} \partial_{j} A_{0} d x,
\end{aligned}
$$

where we used (2)-(4) in the following way:

$$
\begin{gathered}
-\int_{B_{R}} x_{1} F_{21} \operatorname{Im}\left(\bar{\phi} D_{2} \phi\right)+x_{2} F_{12} \operatorname{Im}\left(\bar{\phi} D_{1} \phi\right) d x \\
=\int_{B_{R}} x_{j}|\phi|^{2}\left(\omega+A_{0}\right) \partial_{j} A_{0} d x .
\end{gathered}
$$

Combining (23) and (26), we have from the identity (21)

$$
\begin{aligned}
\int_{B_{R}}(\omega & \left.+A_{0}\right)^{2}|\phi|^{2} d x-\int_{B_{R}} V\left(|\phi|^{2}\right) d x \\
= & \int_{\partial B_{R}} \frac{x_{j} x_{k}}{R} \operatorname{Re}\left(D_{j} \phi \overline{D_{k} \phi}\right)-\frac{R}{2}\left|D_{j} \phi\right|^{2} \\
& -\frac{R}{2} V\left(|\phi|^{2}\right)+\frac{R}{2}\left(\omega+A_{0}\right)^{2}|\phi|^{2} d \sigma_{R} .
\end{aligned}
$$

Thus we have

$$
\begin{aligned}
& \left.\left|\int_{B_{R}}\left(\omega+A_{0}\right)^{2}\right| \phi\right|^{2}-V\left(|\phi|^{2}\right) d x \mid \\
& \leq C \int_{\partial B_{R}} R\left(\left|D_{j} \phi\right|^{2}+\omega^{2}|\phi|^{2}\right. \\
& \left.+\left|A_{0}\right|^{2}|\phi|^{2}+V\left(|\phi|^{2}\right)\right) d \sigma_{R},
\end{aligned}
$$


where $C$ is a positive constant. Considering the Sobolev embedding and the condition of Theorem 1, we know that $\left|D_{j} \phi\right|^{2}, \omega^{2}|\phi|^{2},\left|A_{0}\right|^{2}|\phi|^{2}, V\left(|\phi|^{2}\right) \in L^{1}\left(\mathbb{R}^{2}\right)$. Applying the idea in [16], we know that there exists a sequence $\left\{R_{n}\right\} \rightarrow \infty$ such that

$$
\begin{aligned}
\int_{\partial B_{R_{n}}} & R_{n}\left(\left|D_{j} \phi\right|^{2}+\omega^{2}|\phi|^{2}+\left|A_{0}\right|^{2}|\phi|^{2}+V\left(|\phi|^{2}\right)\right) d \sigma_{R_{n}} \\
& \longrightarrow 0
\end{aligned}
$$

and consequently

$$
\begin{aligned}
\int_{\mathbb{R}^{2}}\left(\omega+A_{0}\right)^{2}|\phi|^{2}-V\left(|\phi|^{2}\right) d x \\
\quad=\lim _{n \rightarrow \infty} \int_{B_{R_{n}}}\left(\omega+A_{0}\right)^{2}|\phi|^{2}-V\left(|\phi|^{2}\right) d x=0 .
\end{aligned}
$$

On the other hand, we know from (1) that

$$
\begin{aligned}
0 & =\int_{\mathbb{R}^{2}} \bar{\phi}\left(-\left(\omega+A_{0}\right)^{2} \phi-D_{j} D_{j} \phi+V^{\prime}\left(|\phi|^{2}\right) \phi\right) d x \\
& =\int_{\mathbb{R}^{2}}-\left(\omega+A_{0}\right)^{2}|\phi|^{2}+\left|D_{j} \phi\right|^{2}+V^{\prime}\left(|\phi|^{2}\right)|\phi|^{2} d x,
\end{aligned}
$$

by taking care of the boundary integral terms as before. Combining (31) and (32), we obtain

$$
\begin{aligned}
\int_{\mathbb{R}^{2}} d\left|D_{j} \phi\right|^{2}+(1-d)\left(\omega+A_{0}\right)^{2}|\phi|^{2} & \\
& +d V^{\prime}\left(|\phi|^{2}\right)|\phi|^{2}-V\left(|\phi|^{2}\right) d x=0,
\end{aligned}
$$

where $0 \leq d \leq 1$ is a constant. We are ready to prove Theorem 1 .

(1) For $0<d \leq 1$, we have $D_{j} \phi \equiv 0$. If $\phi\left(x_{0}\right) \neq 0$, then there exists $\delta>0$ such that $\phi(x) \neq 0$ for $B_{x_{0}}(\delta)=\{x|| x-$ $\left.x_{0} \mid<\delta\right\}$. In the region $B_{x_{0}}(\delta)$, we have $A_{j}=i\left(\partial_{j} \phi / \phi\right)$. Using (4) we have $A_{0}(x)+\omega=0$ in $B_{x_{0}}(\delta)$. On the other hand, from (2) and (3), we deduce that $A_{0}(x)=$ constant $=-\omega$. By (1), we obtain $V^{\prime}\left(|\phi|^{2}\right) \phi=0$ for all $x \in \mathbb{R}^{2}$. By the condition of $V^{\prime}$ and $\phi \in L^{2}$, we conclude that $\phi \equiv 0$.

(2) For $d=0$, we have $V\left(|\phi|^{2}\right)=0$. By the condition of $V$ and $\phi \in L^{2}$, we have $\phi \equiv 0$.

\section{Proof of Theorem 2}

Repeating the similar argument to the proof of Theorem 1, we derive Derrick-Pohozaev type identities for (12)-(15). Suppose that $\left(\phi, A_{0}, A_{1}, A_{2}\right)$ is a solution of (12)-(15). Multiplying (12) by $x_{k} \overline{D_{k} \phi}$ and integrating over $B_{R}$, we obtain

$$
\begin{aligned}
\int_{B_{R}}(\omega & \left.+A_{0}\right) \phi x_{k} \overline{D_{k} \phi} d x-\int_{B_{R}} D_{j} D_{j} \phi x_{k} \overline{D_{k} \phi} d x \\
& +\int_{B_{R}} V^{\prime}\left(|\phi|^{2}\right) \phi x_{k} \overline{D_{k} \phi} d x=0 .
\end{aligned}
$$

Now we set

$$
\begin{gathered}
\mathrm{I}=\int_{B_{R}}\left(\omega+A_{0}\right) \phi x_{k} \overline{D_{k} \phi} d x, \\
\mathrm{II}=\int_{B_{R}} D_{j} D_{j} \phi x_{k} \overline{D_{k} \phi} d x, \\
\mathrm{III}=\int_{B_{R}} V^{\prime}\left(|\phi|^{2}\right) \phi x_{k} \overline{D_{k} \phi} d x .
\end{gathered}
$$

Then, integrating by parts and taking real parts, we have

$$
\begin{aligned}
\operatorname{Re}\{\mathrm{I}\}= & \int_{\partial B_{R}} \frac{R}{2}\left(\omega+A_{0}\right)|\phi|^{2} d \sigma_{x}-\int_{B_{R}}\left(\omega+A_{0}\right)|\phi|^{2} d x \\
& -\int_{B_{R}} \frac{1}{2} x_{j} \partial_{j} A_{0}|\phi|^{2} d x, \\
\operatorname{Re}\{\mathrm{II}\}= & \int_{\partial B_{R}} \frac{x_{j} x_{k}}{R} D_{j} \phi \overline{D_{k} \phi} d \sigma_{R}-\int_{\partial B_{R}} \frac{R}{2}\left|D_{j} \phi\right|^{2} d \sigma_{R} \\
& -\int_{B_{R}} \frac{1}{2} x_{j} \partial_{j} A_{0}|\phi|^{2} d x, \\
\operatorname{Re}\{\mathrm{III}\}= & \int_{\partial B_{R}} \frac{R}{2} V\left(|\phi|^{2}\right) d \sigma_{x}-\int_{B_{R}} V\left(|\phi|^{2}\right) d x .
\end{aligned}
$$

Then we have from the identity (34)

$$
\begin{aligned}
\int_{B_{R}} V & \left(|\phi|^{2}\right)+\left(\omega+A_{0}\right)|\phi|^{2} d x \\
= & \int_{\partial B_{R}}-\frac{x_{j} x_{k}}{R} \operatorname{Re}\left(D_{j} \phi \overline{D_{k} \phi}\right)+\frac{R}{2}\left|D_{j} \phi\right|^{2} \\
& +\frac{R}{2} V\left(|\phi|^{2}\right)+\frac{R}{2}\left(\omega+A_{0}\right)|\phi|^{2} d \sigma_{R} .
\end{aligned}
$$

Applying the same argument in Section 2, the right hand side of the above equality vanishes. Then we conclude that

$$
\int_{\mathbb{R}^{2}} V\left(|\phi|^{2}\right)+\left(\omega+A_{0}\right)|\phi|^{2} d x=0 .
$$

On the other hand, we know from (12)

$$
\int_{\mathbb{R}^{2}}\left(\omega+A_{0}\right)|\phi|^{2}+\left|D_{j} \phi\right|^{2}+V^{\prime}\left(|\phi|^{2}\right)|\phi|^{2} d x=0 .
$$

Combining (38) and (39), we end up with

$$
\int_{\mathbb{R}^{2}}\left|D_{j} \phi\right|^{2}+V^{\prime}\left(|\phi|^{2}\right)|\phi|^{2}-V\left(|\phi|^{2}\right) d x=0 .
$$

Following the reasoning in Theorem 1 , we deduce $\phi \equiv 0$ from the fact $D_{j} \phi \equiv 0$.

For the proof of the second result in Theorem 2, we assume $\phi(x)=u(x) \in \mathbb{R}$. Then (13)-(15) can be rewritten by

$$
\begin{gathered}
\partial_{1} A_{0}=A_{2} u^{2}, \\
\partial_{2} A_{0}=-A_{1} u^{2}, \\
\partial_{1} A_{2}-\partial_{2} A_{1}=-\frac{1}{2} u^{2} .
\end{gathered}
$$


It is easy to check the following identity:

$$
\partial_{1}\left(A_{2} A_{0}\right)-\partial_{2}\left(A_{1} A_{0}\right)=\left(A_{1}^{2}+A_{2}^{2}-\frac{1}{2} A_{0}\right) u^{2}
$$

from which we derive, with the condition $A_{0} \in L^{p}, A_{1}, A_{2} \in$ $L^{q}$ for $1 / p+1 / q=1,2<q<\infty$,

$$
\int_{\mathbb{R}^{2}} \frac{1}{2} A_{0} u^{2} d x=\int_{R^{2}}\left(A_{1}^{2}+A_{2}^{2}\right) u^{2} d x .
$$

Considering $\left|D_{j} u\right|^{2}=|\nabla u|^{2}+\left(A_{1}^{2}+A_{2}^{2}\right) u^{2}$, we have from (38) and (39)

$$
\begin{gathered}
\int_{\mathbb{R}^{2}} V\left(|u|^{2}\right)+\omega|u|^{2}+2\left(A_{1}^{2}+A_{2}^{2}\right) u^{2} d x=0 \\
\int_{\mathbb{R}^{2}}|\nabla u|^{2}+3\left(A_{1}^{2}+A_{2}^{2}\right) u^{2}+\omega|u|^{2}+V^{\prime}\left(|u|^{2}\right)|u|^{2} d x=0 .
\end{gathered}
$$

Then we obtain, for a constant $h \geq 2 / 3$,

$$
\begin{gathered}
\int_{\mathbb{R}^{2}} h|\nabla u|^{2}+(3 h-2)\left(A_{1}^{2}+A_{2}^{2}\right) u^{2}+\omega(h-1)|u|^{2} \\
+h V^{\prime}\left(|u|^{2}\right)|u|^{2}-V\left(|u|^{2}\right) d x=0,
\end{gathered}
$$

which proves the second result in Theorem 2.

\section{Acknowledgments}

The author was supported by Basic Science Research Program through the National Research Foundation of Korea (NRF) funded by the Ministry of Education, Science and Technology (2011-0015866) and also partially supported by the TJ Park Junior Faculty Fellowship.

\section{References}

[1] J. Hong, Y. Kim, and P. Y. Pac, "Multivortex solutions of the abelian Chern-Simons-Higgs theory," Physical Review Letters, vol. 64, no. 19, pp. 2230-2233, 1990.

[2] R. Jackiw and E. J. Weinberg, "Self-dual Chern-Simons vortices," Physical Review Letters, vol. 64, no. 19, pp. 2234-2237, 1990.

[3] D. Chae and O. Yu. Imanuvilov, "The existence of nontopological multivortex solutions in the relativistic self-dual Chern-Simons theory," Communications in Mathematical Physics, vol. 215, no. 1, pp. 119-142, 2000.

[4] H. Chan, C.-C. Fu, and C.-S. Lin, "Non-topological multivortex solutions to the self-dual Chern-Simons-Higgs equation," Communications in Mathematical Physics, vol. 231, no. 2, pp. 189-221, 2002.

[5] K. Choe, "Multiple existence results for the self-dual ChernSimons-Higgs vortex equation," Communications in Partial Differential Equations, vol. 34, no. 10-12, pp. 1465-1507, 2009.

[6] M. Nolasco, "Nontopological $N$-vortex condensates for the self-dual Chern-Simons theory," Communications on Pure and Applied Mathematics, vol. 56, no. 12, pp. 1752-1780, 2003.

[7] R. Wang, "The existence of Chern-Simons vortices," Communications in Mathematical Physics, vol. 137, no. 3, pp. 587-597, 1991.
[8] R. M. Chen and D. Spirn, "Symmetric Chern-Simons-Higgs vortices," Communications in Mathematical Physics, vol. 285, no. 3, pp. 1005-1031, 2009.

[9] J. Han and N. Kim, "Nonself-dual Chern-Simons and MaxwellChern-Simons vortices on bounded domains," Journal of Functional Analysis, vol. 221, no. 1, pp. 167-204, 2005.

[10] J. Han and N. Kim, "Corrigendum: nonself-dual Chern-Simons and Maxwell-Chern-Simons vortices on bounded domains," Journal of Functional Analysis, vol. 242, no. 2, p. 674, 2007.

[11] R. Jackiw and S.-Y. Pi, "Classical and quantal nonrelativistic Chern-Simons theory," Physical Review, vol. 42, no. 10, pp. 3500-3513, 1990.

[12] G. V. Dunne, Self-Dual Chern-Simons Theories, 1995.

[13] P. A. Horvathy and P. Zhang, "Vortices in (abelian) ChernSimons gauge theory," Physics Reports, vol. 481, no. 5-6, pp. 83142, 2009.

[14] J. Byeon, H. Huh, and J. Seok, "Standing waves of nonlinear Schrödinger equations with the gauge field," Journal of Functional Analysis, vol. 263, no. 6, pp. 1575-1608, 2012.

[15] H. Huh, "Standing waves of the Schrodinger equation coupled with the Chern-Simons gauge field," Journal of Mathematical Physics, vol. 53, no. 6, Article ID 063702, 8 pages, 2012.

[16] H. Berestycki and P.-L. Lions, "Nonlinear scalar field equations. I. Existence of a ground state," Archive for Rational Mechanics and Analysis, vol. 82, no. 4, pp. 313-345, 1983. 


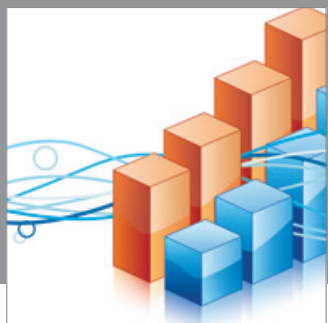

Advances in

Operations Research

mansans

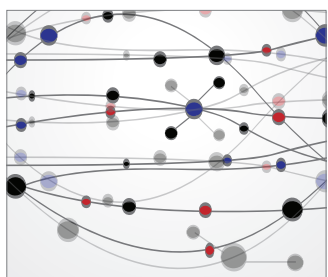

The Scientific World Journal
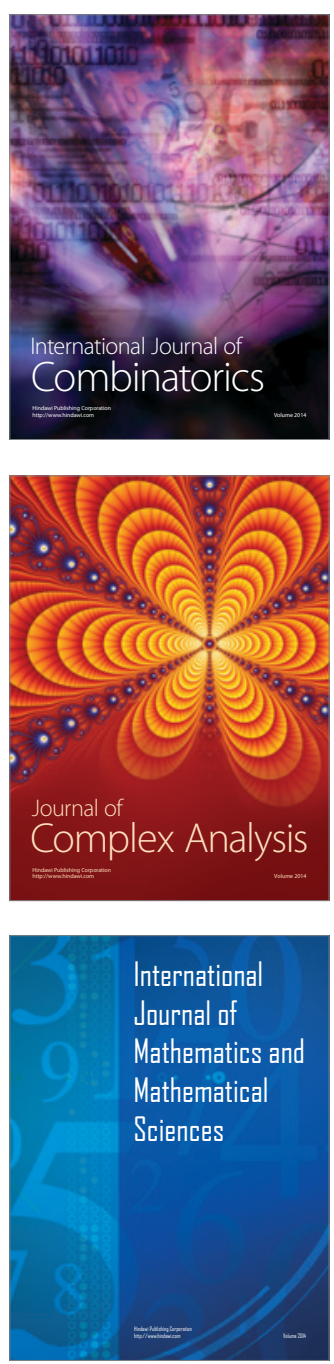
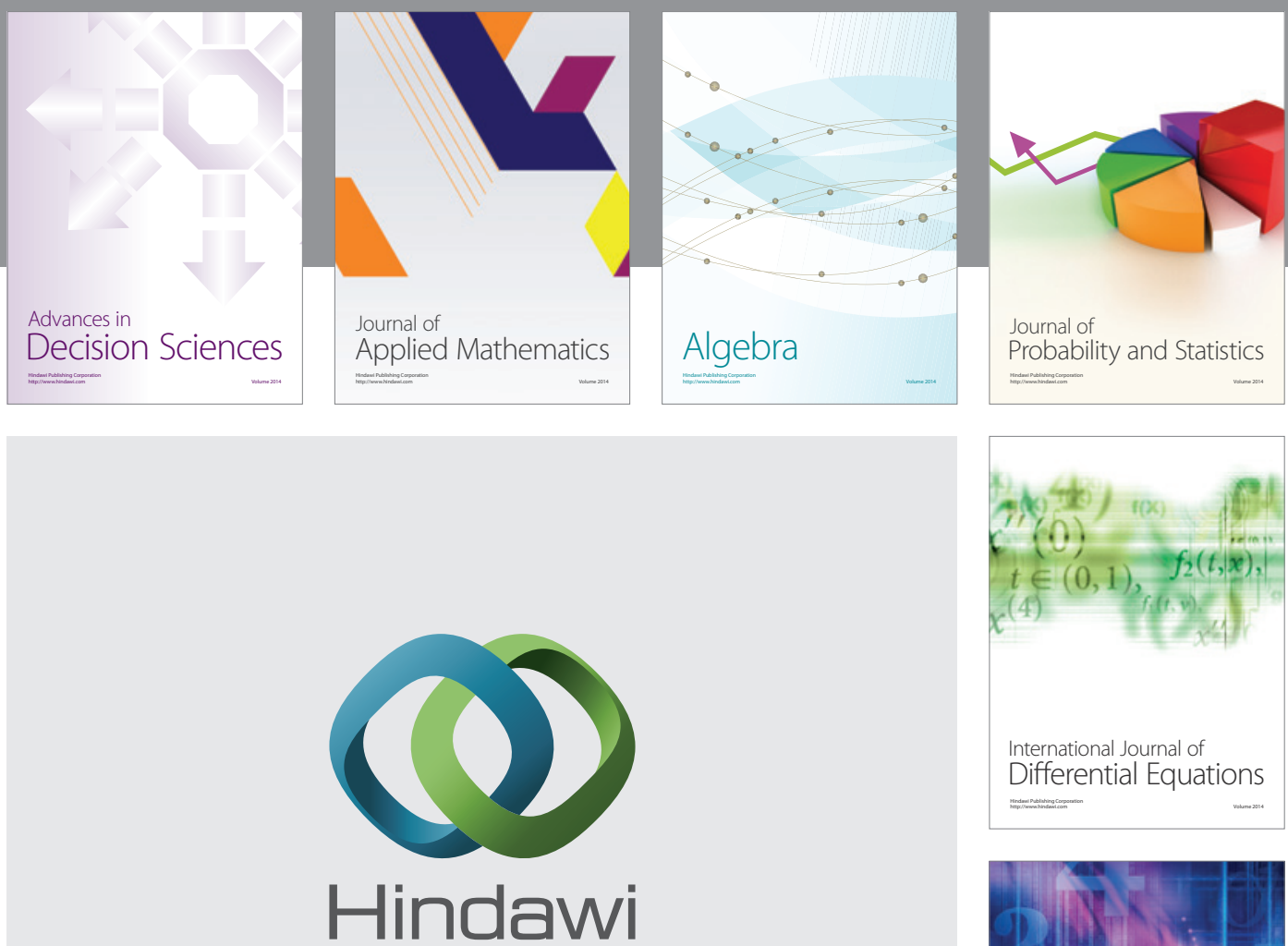

Submit your manuscripts at http://www.hindawi.com
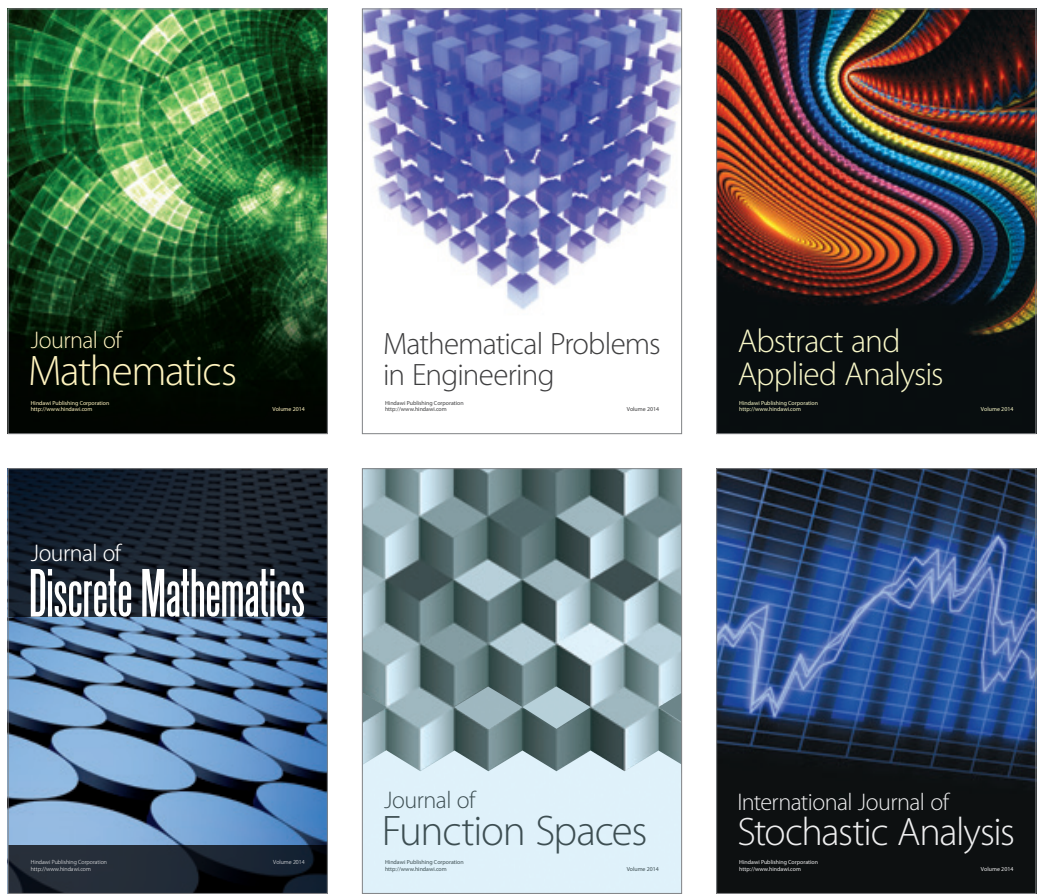

Journal of

Function Spaces

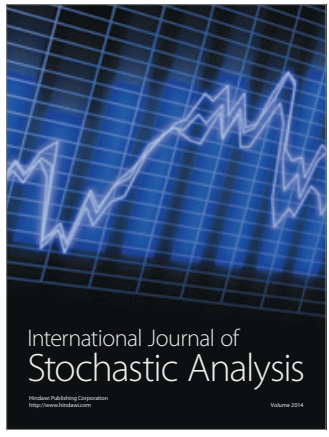

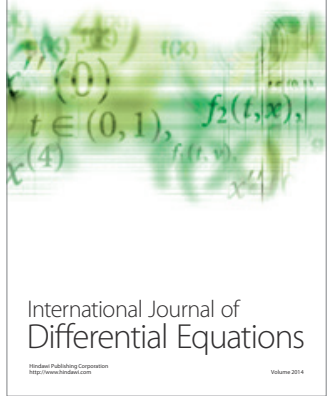
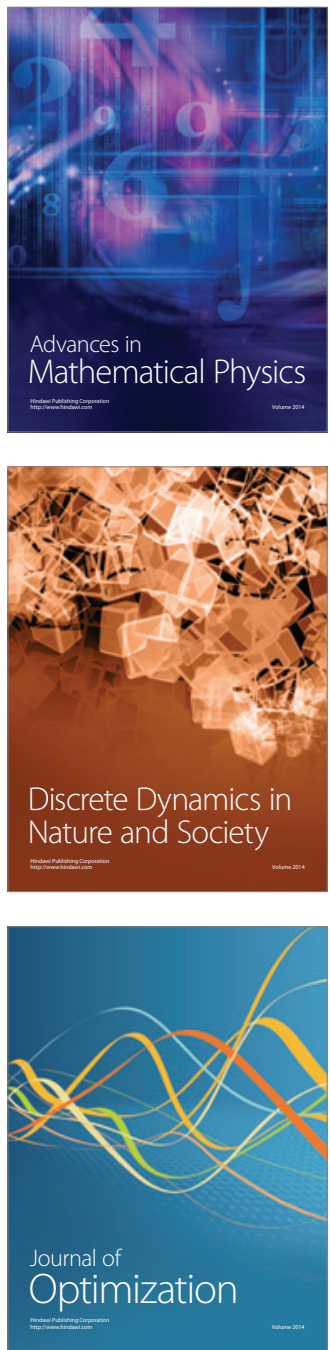